\title{
Une information ciblée qui améliore la vie - et augmente les primes...
}

Il y a quelques mois, les panneaux d'affichage nous interpellaient sur le thème de la migraine. Qui se sentait concerné était invité à consulter un médecin. Pourquoi? Pfizer connaît la réponse.

On a vu encore, sur tous les murs et dans de nombreux lieux d'aisance, des sièges de toilette parmi les fauteuils rouges d'une salle de spectacle. On fait là allusion à un symptôme courant: la vessie hyperactive. Les passants ne savent pas ce que c'est. On leur suggère de consulter leur médecin. Pourquoi? Pfizer offre aujourd'hui le médicament sans doute le mieux documenté pour le traitement symptomatique des véritables problèmes de vessie irritable.

Des recommandations fleurissent aussi sur tous les murs proposant une consultation médicale pour résoudre les problèmes d'érection. C'est l'homme qui décide si son érection est faible ou non, mais pour tirer la chose au clair, il lui faudra consulter.
Au cabinet du médecin, il demandera bien sûr avec insistance une ordonnance pour du Viagra. Merci Pfizer!

Quant à savoir comment le Viagra, la vessie hyperactive et la migraine peuvent être liés sur le plan clinique et faire ainsi grimper les primes d'assurance, je préfère ne pas vous le dévoiler, pour rester dans l'esprit des publicitaires d'aujourd'hui. Mais vous l'avez sûrement compris, non? Autrement, demandez ... ben, euh ... à votre représentant en produits pharmaceutiques!

Vous en saurez plus sur ce mode improbable de promotion de la santé en lisant l'article de Hollon MF. Direct-to-consumer advertising: a haphazard approach to health promotion. JAMA 2005;293: 2030-3.

Dr Max Giger, membre du Comité central de la FMH 